\title{
Experimental Demonstration of Cost-Effective Intensity-Modulation and Direct-Detection Optical Fast-OFDM over 40km SMF Transmission
}

\author{
E. Giacoumidis ${ }^{1}$, S.K. Ibrahim ${ }^{2,3}$, J. Zhao ${ }^{2}$, J.M. Tang ${ }^{4}$, I. Tomkos ${ }^{1}$, and A.D. Ellis ${ }^{2}$ \\ ${ }^{1}$ Athens Information Technology (AIT)/Networks and Optical Communications (NOC) Group, Athens, Greece \\ ${ }^{2}$ Photonic Systems Group, Tyndall National Institute and Department of Physics, University College Cork, Ireland \\ ${ }^{3}$ Now with: Intune Networks, 9 C Beckett Way, Park West, Dublin 12, Ireland \\ ${ }^{4}$ Bangor University/School of Electronic Engineering, Bangor, Wales, UK \\ E-mail: igia@ait.gr
}

\begin{abstract}
We demonstrate the first experimental implementation of intensity-modulation and direct-detection 7.6Gb/s DBPSK-based DSB optical Fast-OFDM with a reduced subcarrier spacing equal to half of the symbol rate per subcarrier over $40 \mathrm{~km} \mathrm{SMF}$. OCIS codes: (060.2330) Fiber optics communications; (060.4080) Modulation; (060.4510) Optical communications
\end{abstract}

\section{Introduction}

Optical fast orthogonal frequency division multiplexing (FOFDM) is a promising candidate for digital signal processing (DSP) based modern communication systems, and has attracted great research interest due to its improved spectral efficiency and reduced system complexity [1-6].

FOFDM, different from conventional OFDM, uses only half of the conventional OFDM subcarrier spacing and simple single-quadrature modulation formats. The subcarrier multiplexing/demultiplexing in optical FOFDM can be implemented by a discrete cosine transform (DCT) [1] or discrete-Fourier transform (DFT) [2]. Recently, this technique has been experimentally demonstrated in single-mode fibre (SMF)based optical communication systems using coherent detection $[3,4]$, where it exhibits enhanced tolerance to frequency offset and in-phase/quadrature imbalance [1-4] when compared to conventional OFDM. However, coherent detection is expensive. On the other hand, direct-detection (DD) significantly reduces the system complexity. Intensity-modulation and DD (IM/DD) OFDM has been considered as one of the promising candidates for cost-sensitive applications including Ethernet, access and short metro networks [1-6]. FOFDM has been investigated for installed legacy MMF-based links and Ethernet backbones [5] using the same cost effective IM/DD configuration.

To our knowledge, experimental demonstrations of FOFDM over SMF links using IM/DD have not been reported. In this paper, we experimentally demonstrate the world first differential binary phase shift keying (DBPSK)-encoded double-side-band (DSB) optical FOFDM system over 40m of SMF using $\mathrm{IM} / \mathrm{DD}$ with a net data rate of $7.6 \mathrm{~Gb} / \mathrm{s}$ (excluding cyclic prefix $[\mathrm{CP}]$ and $7 \%$ FEC overhead), for a targeted bit-error-rate (BER) of $10^{-3}$.

\section{Experimental setup}

Fig. 1 shows the experimental setup of DBPSK-based DSB optical FOFDM system. The FOFDM signal generation and decoding blocks were implemented offline using MATLAB. The transmitted FOFDM data signals were generated by implementing serial-to-parallel conversion, single-dimensional mapping (DBPSK modulation), 64-point inverse DCT (IDCT), addition of 25\% CP and parallel-to-serial conversion. The first subcarrier (DC) was not modulated, allowing for AC-coupled driving amplifiers and receivers while 27 subcarriers (SC\#1-SC\#27) were used for data transmission. The output of the IDCT is real-valued with a DSB spectrum and the generated samples were downloaded to an arbitrary waveform generator (AWG). The AWG operated in the interleaved mode with a sampling rate of $24 \mathrm{GS} / \mathrm{s}$ and a resolution of 8 bits. The 3-dB analogue bandwidth of the AWG, excluding the DAC sinc-function roll-off, is around $8 \mathrm{GHz}$. The nominal signal line data rate including the CP, FEC overhead was around $10.125 \mathrm{~Gb} / \mathrm{s}$ $(24 \times 27 / 64)$. The net bit rate was $8.1 \mathrm{~Gb} / \mathrm{s}$ when accounting for the $\mathrm{CP}$, and $7.6 \mathrm{~Gb} / \mathrm{s}$ when excluding the $\mathrm{CP}$ and 7\% FEC overhead. A zero-chirp Mach-Zehnder modulator (MZM) biased around the quadrature point was used to modulate the optical carrier with the electrically generated FOFDM signal to enable DD, while the electrical peak-to-peak voltage into the MZM was $0.5 \mathrm{Vpi}$. The generated optical FOFDM signal at the output of the MZM was amplified 1.3dBm using an erbium doped fibre amplifier (EDFA). The use of the 
EDFA and the associated $0.3 \mathrm{~nm}$ bandpass filter (BPF) for amplified spontaneous emission noise suppression was only due to the low output power of the MZM and could be omitted in practice provided that a high power laser could be used. A variable optical attenuator (VOA) was used to vary the input power to the photodiode. The received FOFDM signal was then converted into the electrical domain using a $9 \mathrm{GHz}$ PIN photo-receiver with an integrated transimpedance amplifier (TIA).

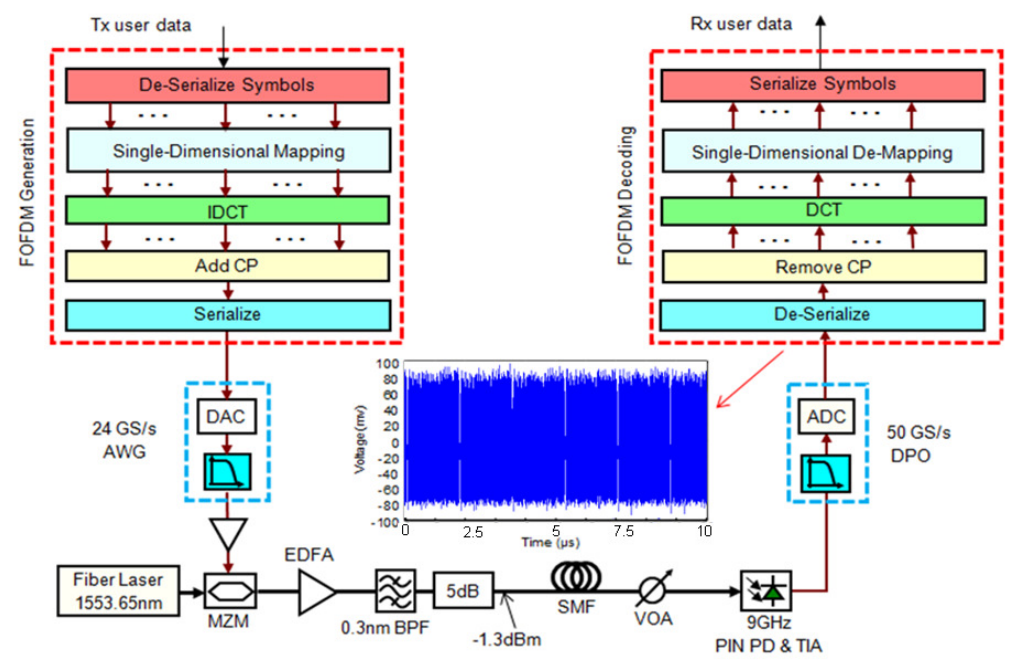

Fig. 1. Experimental SMF-based IM/DD FOFDM setup.

The received electrical FOFDM signal was captured using a 50GS/s (16GHz 3-dB bandwidth) real-time digital phosphor oscilloscope (DPO) and was processed offline using MATLAB to decode the received FOFDM signals. The received 50GS/s signals were down-sampled to $24 \mathrm{GS} / \mathrm{s}$ before been processed by the FOFDM decoding block which consists of serial-to-parallel conversion, CP removal, 64-point DCT, singledimensional de-mapping (differential detection), and parallel-to-serial conversion. The received FOFDM data was captured over 10 $\mu$ s duration as shown in the time domain trace in Fig. 1. The BER measurement was over 67,500 data bits using direct error counting.
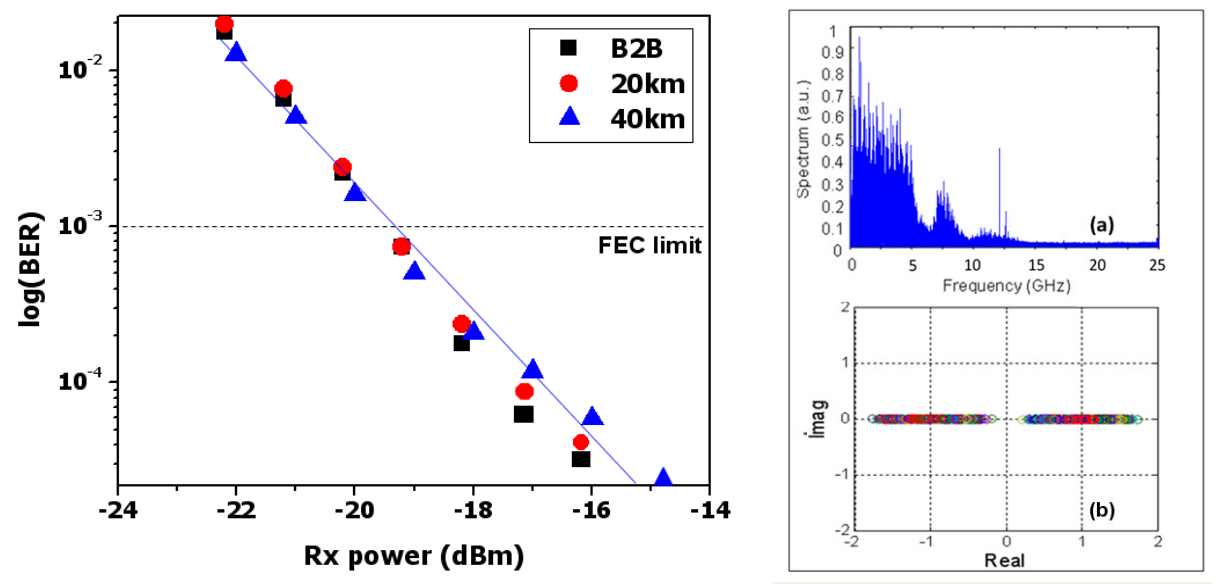

Fig. 2. Left: BER curves for the received DBPSK FOFDM signal for B2B (black squares), over 20km (red circles), and over 40km SMF (blue triangles). Right: (a) Optical spectrum output of the SMF measured at a received power of $11 \mathrm{dBm}$ over $40 \mathrm{~km}$ for error-free operation. (b) Recovered error-free constellation diagram of DBPSK FOFDM at a received power of $-11 \mathrm{dBm}$ for $40 \mathrm{~km}$ transmission.

\section{Results}

Fig. 2 (left) shows the measured BER performance of the 7.6Gb/s DBPSK-based DSB FOFDM signal for the back-to-back (B2B) case and after transmission of 20/40km SMF-link. The required received optical 
power at a BER of $10^{-3}$ was measured to be $\sim-19.9 \mathrm{dBm}, \sim-19.7 \mathrm{dBm}$, and $\sim-19.2 \mathrm{dBm}$ for B2B, $20 \mathrm{~km}$ and $40 \mathrm{~km}$ of SMF transmission, respectively, leading to a maximum power penalty of only $\sim 0.7 \mathrm{~dB}$ corresponding to the case of 40km SMF-link. Fig. 2 (right [a]) shows the optical spectrum output of the SMF measured at a received power of $-11 \mathrm{dBm}$ over $40 \mathrm{~km}$ corresponding to error-free operation; where the frequency range of $\sim 6.25 \mathrm{GHz}-12.5 \mathrm{GHz}$ contains the complex conjugate data generated by Hermitian symmetry. Fig. 2 (right $[\mathrm{b}]$ ) depicts the corresponding recovered constellation diagram of the DBPSK FOFDM. Fig. 3 (left [b]) shows the optical spectrum of the FOFDM signal at the output of the $0.3 \mathrm{~nm}$ BPF.

In Fig. 3, a comparison between DBPSK FOFDM (experimental) and 4QAM conventional OFDM (theoretical) is illustrated, revealing that similar spectral efficiency and receiver sensitivity can be achieved. Nevertheless, the simpler carrier phase recovery and reduced complexity of DBPSK FOFDM assigns a benefit over 4QAM OFDM. Note that in the case of the conventional OFDM, similar procedures to the FOFDM are adopted by replacing the DCT block with a DFT. The calculated 4QAM DSB OFDM spectrum at the output of the PIN-detector is depicted in Fig. 3 (left [a]).
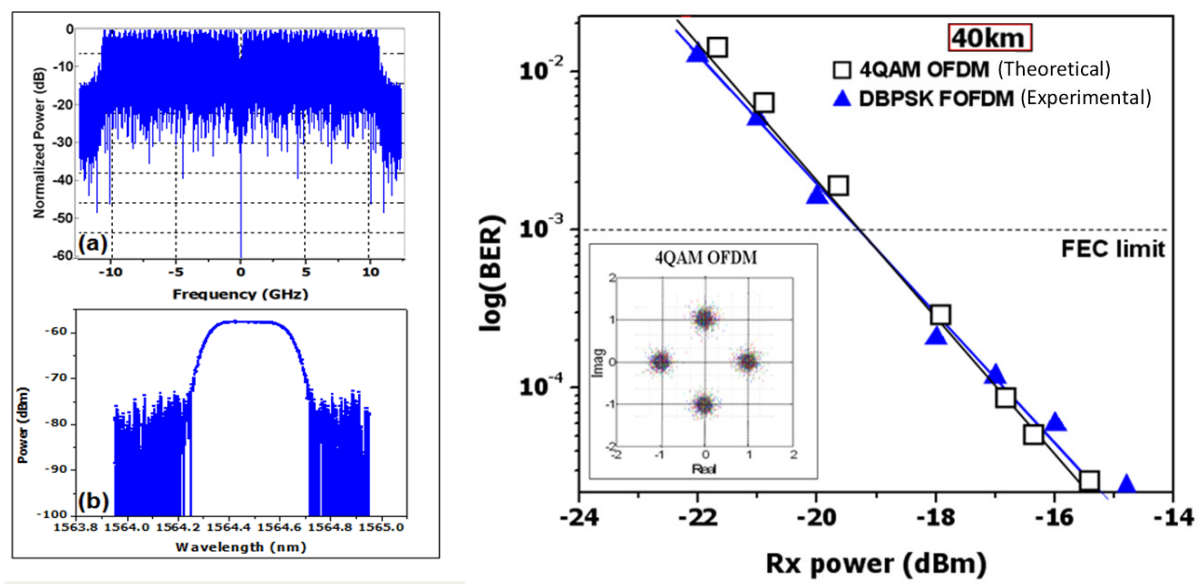

Fig. 3. Left: (a) 4QAM OFDM spectrum (theoretical) at the output of the PIN-detector and (b) optical spectrum of the DBPSK FOFDM signal (experimental) output of the $0.3 \mathrm{~nm}$ BPF. Right: BER curve comparison for the received signal between 4QAM OFDM (theoretical) and DBPSK FOFDM (experimental) at 7.6Gb/s after 40km SMF transmission. Inset: the theoretically recovered 4QAM OFDM constellation diagram at $-18 \mathrm{dBm}$ received power.

\section{Conclusions}

We have experimentally demonstrated for the first time to our knowledge an optical DBPSK-based IM/DD DSB FOFDM system with a subcarrier spacing equal to half of the symbol rate per subcarrier operating at a net data rate of $7.6 \mathrm{~Gb} / \mathrm{s}$ over $40 \mathrm{~km}$ of SMF. The receiver maximum sensitivity penalty at $10^{-3}$ was measured to be only $\sim 0.7 \mathrm{~dB}$ for $40 \mathrm{~km}$ SMF transmission when compared to the B2B case. Due to its simple carrier phase recovery and reduced complexity, DBPSK FOFDM is preferred to a 4QAM conventional OFDM system having similar spectral efficiency and receiver sensitivity. This paper demonstrates the feasibility of implementing DBPSK-based IM/DD DSB FOFDM in SMF links for access networks enabling higher speed transmission.

This work was supported by Science Foundation Ireland (SFI) under grant number 06/IN/I969, and EU funded projects EUROFOS, MODE-GAP and ACCORDANCE.

\section{References}

[1] J. Zhao and A.D. Ellis, "A novel optical fast OFDM with reduced channel spacing equal to half of the symbol rate per carrier," in Proc. OFC'2010, paper OMR1.

[2] J. Zhao and A.D. Ellis, "Discrete-Fourier transform based implementation for optical fast OFDM," in Proc. ECOC'2010, paper Tu.4.A.3.

[3] S.K. Ibrahim, J. Zhao, D. Rafique, J.A. O’Dowd, A.D. Ellis, "Demonstration of World-First Experimental Optical Fast OFDM System at 7.174Gbit/s and 14.348Gbit/s," in Proc. ECOC'2010, post-deadline paper PD3.4.

[4] J. Zhao, S.K. Ibrahim, D. Rafique, P. Gunning, A.D. Ellis, "Symbol synchronization exploiting the symmetric property in optical fast OFDM," IEEE Photonics Technology Letters, vol. 23, pp. 594-596, 2011.

[5] E. Giacoumidis, I. Tomkos, J.M. Tang, “A Performance of Optical Fast-OFDM in MMF-Based Links," in Proc. OFC'2011, paper OWU3.

[6] C. Lei, H. Chen, M. Chen, and S. Xie, "16×10Gb/s symmetric WDM-FOFDM-PON realization with colorless ONUs," Opt. Express 19, pp. 15275-15280, 2011. 